\title{
Uncertainty Analysis and Application of Construction Diversion Tunnel Discharge Capacity
}

\author{
Wang Changhong* \\ School of Civil Engineering and Transportation, \\ South China University of Technology; \\ Guangzhou 510640; China; \\ chhwang1@scut.edu.cn
}

\author{
Wang Zhaoli \\ School of Civil Engineering and Transportation, \\ South China University of Technology; \\ Guangzhou 510640; China; \\ wangzhl@scut.edu.cn
}

\begin{abstract}
For the construction of the diversion tunnel discharge capacity problem, the difference of the starting point between the theory of uncertainty and the traditional deterministic theory is analyzed. The discharge capacity is affected by a comprehensive variety of factors, including the cross section area, roughness, wetted perimeter, Chézy coefficient and many other factors, and thus discharge capacity is multiple functions of these random variables. On the basis of the actual in-depth research and getting a lot of information, reasonable statistical analysis and properly determination of the distribution of various types of random variables are made. Establishing a reasonable probability density function and performance function, using Monte-Carlo method to simulate, the more objective and real discharge capacity reliability of the construction of the diversion tunnel can be accessed. Combined with practical engineering examples under the actual monitoring data for comparison, the results are objective and reasonable. The research results can provides a certain degree of programming reference for the same type for the future diversion project of the the same river basin.
\end{abstract}

Keywords-discharge capacity; reliability; Uncertainty; diversion tunnel; Monte-Carlo simulation method

\section{INTRODUCTION}

Traditional deterministic theory insist that as long as the quality of the project in line with the design and construction requirements, then the actual discharge capacity of the construction diversion tunnel at the design condition should be equal to of the design value. Nevertheless uncertainty theory believes that the actual discharge capacity of the construction diversion tunnel are not the only values and not deterministic; a certain guarantee rate also known as reliability are rather linked. Compared with the deterministic theory, the latter is more in line with the objective conditions of the project.

Specifically, the discharge capacity is affected by a comprehensive variety of factors, including the cross section area, roughness, wetted perimeter, Chézy coefficient and many other factors, and thus discharge capacity is multiple functions of these random variables ${ }^{[1]}$. Such as roughness $n$, is an integrated random variables reflecting the flow resistance of diversion structures, including both over-flow surface roughness, but also other aspects of the flow regime of factors, and these factors are random; Thus, roughness $n$ is itself functions as a multivariate random variable or random function, other factors affecting the diversion flow capacity is also similar. Therefore, the reliability issues with discharge capacity of diversion structures are considering the assurance of the design discharge rate ${ }^{[1]}$ based on the effect of these random variables.

\section{RELIABILITY DEFINITION \& ANALYSIS OF RELIABILITY PROBLEMS}

\section{A. Reliability definition of engineering systems}

System reliability can be defined as: within the system at a given time $t$, the probability of complete scheduling capabilities under the conditions stipulated ${ }^{[2-5]}$, Commonly risk $P_{f}$ and reliability index $\beta$ measure reliability in quantitative analysis.

If $R$ represents a generalized resistance and $S$ represents the generalized load of the structure, limit state of the structure is expressed as $Z=R-S=0$ Then $Z>0$, the structure is in reliable state; $Z<0$, the structure is in failure state 。

According to the definition of reliability and the principle of probability theory, if the random variable vectors $X=\left(X_{1}, X_{2} \ldots, X_{3}\right)$ of the system is composed of by the basic random variable, the corresponding probability density function $\mathrm{f}(\mathrm{X})$, whereby the function represented by this random vector $Z=g(X)$ is called the function;

Performance function is a function that represents the System State, working conditions can be expressed as:

$$
Z=g(X)\left\{\begin{array}{cc}
<0 & \text { failure state } \\
=0 & \text { limit state } \\
>0 & \text { reliable state }
\end{array}\right.
$$

The probability that the risk of system failure rate is:

$$
P_{f}=p(Z<0)=\int_{F} f(x) d x
$$

In this formula : $F=\{x \mid g(x)<0\}$ is failure domain。 
During the study of the diversion tunnel discharge capacity in cofferdam conditions, the diversion design flow $Q_{d}$ as a determined targeting flow, According to equation (1), the basic expressions of discharge risk of diversion tunnel can be established of, the following functions is:

$$
Z=Q_{d}-Q
$$

Here in: $Q_{d}$ is diversion design flow, $\mathrm{m}^{3} / \mathrm{s} ; \mathrm{Q}$ is the actual discharge for tunnel, $\mathrm{m}^{3} / \mathrm{s}$ 。

Obviously, when $Z \geq 0$, the system is in safe or critical state; when $Z<0$, the system is in dangerous or detrimental state. Therefore, the risk rate of diversion tunnel discharge calculation is to determine the probability of an event when the case occurring of $Z<0$.

\section{B. Analysis of reliability problems}

The core issue of reliability analysis is to calculate the random information processing and stochastic risk. When normal distribution factors affecting the reliability and performance function as a linear function of time, the risk rate and reliability can be accurately calculated; But in practice many of the basic random variables are not normally distributed, and functions are mostly nonlinear functions, therefore a simple method can not calculate the reliability directly and accurately. Commonly used approximation methods including: Center point method, checking point method (JC method) and mapping transformation method ${ }^{[6]}$, these analytical methods are established based on the theory of probability and numerical analysis.

Specifically to calculate the risk of diversion tunnel discharge rate, using the analytical method firstly to analyze the actual discharged $Q$, getting probability distribution (function) situation, then probability distribution functions on the risk of the diversion tunnel overflow by means of mathematical analysis can be got .This approach requires knowledge on quantitative distribution characteristics of $Q$, due to the complexity of the hydraulic distribution uncertainties, the probability distribution of $Q$ is not easy or difficult to get, Wanting to get analytical solution is very difficult, so as to affect the risk of diversion tunnel discharge rate calculation, including the impact on the accuracy of the results.

Analytic method by using numerical simulation methods can overcome the "bottleneck" problems, this paper will focus on the method of numerical simulation.

Representative methods of numerical simulation, such as Monte-Carlo(MC) its basic idea is to construct the probabilistic model of random variables and probability sampling simulation model, when the simulating count is large enough, simulation results can be used as approximate solution of the original problem, and meet certain accuracy requirements. Although there are deficiencies of analog computational complexity of this method, but in case of better hardware and justified algorithms, it is an ideal way to gain reliability.

Through multiple simulations of the diversion tunnel outlet flow $\mathrm{Q}$, getting computation and statistical function result, diversion tunnel failure events probability or discharge risk rate can be obtained.

\section{MATHEMATICAL MODEL OF RISK OF DIVERSION TUNNEL DISCHARGE RATE}

\section{A. Establishment of the function of discharge risk rate}

Because of discharge flow of diversion tunnel showing a alternation of pressurized 、 semi-pressurized and non-pressure in the flood, in order to research its reliability problem of discharge capacity $Q$ of the diversion, examining the adverse flow patterns, that is pressure flow pattern. The tunnel pressurized discharge capacity stream flow $Q$ (here ignoring the frictional head loss) can be expressed as:

$$
Q=\mu A \sqrt{2 g\left(H_{0}+i L-\eta d\right)}
$$

Here in: $\quad \mu=\frac{1}{\sqrt{1+\sum \xi+\frac{2 \mathrm{gL}}{\mathrm{C}^{2} \mathrm{R}}}}, \quad H_{0}$ is water depth above the tunnel inlet floor,m;

$\sum \xi$ is the sum of three local loss coefficient including the imports section 、 the exit section and center section;

$R$ is hydraulic radius, $\mathrm{m} ; C$ is the Chézy coefficient $\mathrm{C}=$ $\frac{1}{\mathrm{n}} \mathrm{R}^{1 / 6} ; A$ is tunnel section area, $\mathrm{m}^{2} ; \mathrm{G}$ is gravitational acceleration, $9.81 \mathrm{~m} / \mathrm{s}^{2} ; h$ is imports and exports rising of the tunnel, $\mathrm{m} ; i$ is tunnel slope rate; $L$ is tunnel length, $m ; \mathrm{n}$ is roughness rate。

\section{B. Uncertainty analysis of the effect of the diversion tunnel discharge capacity}

Then considering uncertainty effects of the parameters: in equation (4), $X=(A, R, n, h, i, L)$ is a random vector reflecting the discharge flow ability of diversion tunnel, function $Q$ is expressed as a function of the random vector, namely $Q=f(A, R, n, h, i, L)$. The uncertainty of $A, R, n, h, i, L$ affects the mean and variance of $Q$, following a simple analysis of uncertainties of hydraulic factors:

\section{1) As for hydraulic structures, the hydraulic elements:}

such as tunnel section area $A$, the hydraulic radius $R$, tunnel slope rate $i$, tunnel length $L$, on the one hand the uncertainties of such hydraulic elements are caused by measurement, construction materials and lofting error; on the other hand due to flow cavitation and riverbed scour change. Here the former reason is only taken into account. This pure geometry (measurement, lofting) error, mathematically is proven to be approximately normally distributed ${ }^{[7]}$.

2) Randomness of roughness $n$ :

arising primarily from the construction and operation of the uncertain and irregular changes in the structure of the tunnel wall and, in addition, cavitation phenomena and factors such as depth changes also have different effects on $\mathrm{n}$, it is difficult to determine rigorously the distribution of $\mathrm{n}$ approximation can only be made:

Applying stochastic simulation and assumed distribution method, scholars study of the distribution ${ }^{[8]}$ of roughness coefficient $\mathrm{n}$. Based on the information of $n$ values. given the normal value $n_{b}$, minimum value $n_{a}$ and maximum value $n_{c}$, and then assuming $\mathrm{n}$ approximately triangular distribution is feasible.

3) Randomness of $h$ (the upstream and downstream water level difference):

on one hand comes from the uncertainty of water level (the factors influencing the level are complex), on the other 
hand comes from construction and measurement error; These factors can be considered independent by each other, the influence is difficult to distinguish between primary and secondary, so they are treated as approximately normally distributed ${ }^{[9]}$.

These general parameters of hydraulic distributions of random variables can be determined in this way: assuming that design values is reliable, taking design values for random variables mean, then average error of construction or measurement can be used to acquire mean-square deviation $^{[9]}$.

Such as $\delta_{L}$ is construction error of tunnel length $L$, That is $\delta_{L}=L-E(L)$, tunnel length $L$ is Postulated in normal distribution, in the probability theory, we know that $\delta_{L}$ is also normally distributed, the expectations of $\delta_{L}$ is $E\left(\delta_{L}\right)=0$, the mean-square deviation of $\delta_{L}$ is $\sigma_{\delta_{L}}=\sigma_{L}$, the average error is ${ }^{[1]}$ :

$$
\delta_{L}=\int_{-\infty}^{+\infty}\left|\delta_{L}\right| f\left(\delta_{L}\right) d \delta_{L}
$$

Getting the quadrature of formula (5), and noting $\sigma_{\delta_{L}}=\sigma_{L}$, getting the following equation:

$$
\sigma_{L}=\sigma_{\delta_{L}}=\sqrt{\frac{\pi}{2}} \bar{\delta}_{L}
$$

With the estimate of average error $\overline{\delta_{L}}$, then by equation (6) to seek mean-square deviation of random variable and probability distribution functions of tunnel length $L$ can be obtained.

Similarly, other random variables $A 、 R 、 i$ and $h$, assuming that they are normally distributed, taking its design values as mean value, following the estimates of their errors, using similar to equation (6) formula, there will be mean-square deviation, then their probability distribution function expressions can be obtained.

For roughness $\mathrm{n}$, where assuming the triangular distribution, triangular probability density function $f(x)$ and its distribution function $F(x)$ is:

$$
f(x)= \begin{cases}\frac{2(x-a)}{(b-a)(c-a)} & a \leq x \leq b \\ \frac{2(c-x)}{(c-a)(c-b)} & b<x \leq c \\ 0 \quad x<a, & \text { or } x>c\end{cases}
$$

$$
F(x)=\left\{\begin{array}{cc}
0 & x<a \\
\frac{(x-a)^{2}}{(b-a)(c-a)} & a \leq x \leq b \\
1-\frac{(c-x)^{2}}{(c-b)(c-a)} & b<x \leq c
\end{array}\right.
$$

Taking the design value for the mean value of roughness $n$, denoted $E(n)$, and then estimating $n$ for its maximum value $n_{c}$ and minimum value of $n_{a}$, you can get three triangular distribution parameters of roughness $n: a, b$ and $c$, resulting in expression of the probability distribution function.

\section{Simulation process}

\section{1) Pseudo random number generating}

The program performed by $M C$, large amounts of random numbers need to be generated firstly; Uniformly distributed pseudo-random numbers is the basis for analoging random number of another distributions, there are algorithm methods, such as cache method, iterative method, method of residues and shifting method etc, this paper is using random number generator program ${ }^{[1,10]}$ recommended by related literature, it is verified with better uniformity and independence, and has sufficiently long periods of time.

\section{2) Each random variable sample of effecting discharge capacity of diversion tunnel}

a) Normal distribution random number simulation method

$n$ random samples of the uniform distribution $r_{1}, r_{2} \ldots, r_{n}$ are generated in $[0,1]$. On the basis of them, the random number $r_{i}$ is treated as the probability of an event occurring, the sampled value of the random variable $X$ at a given distribution is calculated. For a normal random variable can be generated using the following method:

$$
\begin{aligned}
& u_{1}=\sqrt{-2 \ln r_{1}} \cos \left(2 \pi r_{2}\right) \\
& u_{2}=\sqrt{-2 \ln r_{1}} \sin \left(2 \pi r_{2}\right)
\end{aligned}
$$

$u_{1}, u_{2}$ are independent of each other, and obedience $N[0,1]$, thus $u_{1}, u_{2}$ can be treated as two independent standard normally distributed random variable samples; random variables $x_{1}, x_{2}$ of normal distribution $N\left[u, \sigma^{2}\right]$ can be obtained by linear transformations:

$$
\left\{\begin{array}{l}
x_{1}=u_{1} \sigma+u \\
x_{2}=u_{2} \sigma+u
\end{array}\right.
$$

b) simulation method of other distributed random number

For other types of distributions, when generating random variables that are required, the commonly used method is the inverse function of probability distribution function must be exported, this is very difficult for some complex distribution; Thus it applies only to simple distribution, specific practices are as follows:

Let $X$ be random variables and be subject to continuously distributed, whose distribution function is $F_{x}(x)$; Given $F_{x}(x)=r$, corresponding to $r$ the value $x$ is:

$$
X=F_{x}^{-1}(r)
$$

In the formula: $F_{x}^{-1}(\bullet)$ is inverse functions of $F_{x}(\bullet)$;

When $r$ is uniformly distributed random number in $[0,1]$ made on machine, the value $x$ calculated by equation (12) is the random variable samples of $X$.

Application of inverse transformation method, the random variable of uniform distribution, exponential distribution and triangular distribution can easily be got.

Due to the roughness coefficient $\mathrm{n}$ following triangular distribution, generation of random variables can be used with the inverse transform method, Assuming $r_{l}$ as random number in $U[0,1]$, then

$$
x_{1}= \begin{cases}a+\sqrt{(b-a)(c-a) r_{i}} & a \leqslant x_{i} \leqslant b \\ c-\sqrt{\left(1-r_{i}\right)(c-b)(c-a)} & b<x_{i} \leqslant c\end{cases}
$$

\section{Calculation of the risk}

The sample value for each random variable is substituted to equation (4), then calculating $Q_{i}$ and comparing $Q_{i}$ and $Q_{d}$. if $Q_{i}<Q_{d}$, Meaning the failure of 
statistical modellin, assuming total number of simulation to be $N$, number of failures to be $N_{f}$, Therefore, the estimated value for $P_{f}$, i.e. failure probability of diversion tunnel discharge capacity is :

$$
\tilde{P}_{f}=\frac{N_{f}}{N}
$$

From Bernoulli's law of large numbers:

$$
\lim _{N \rightarrow+\infty} P\left[\left|\frac{N_{f}}{N}-P_{f}\right|<\varepsilon\right]=1
$$

From the above formula: $\tilde{P}_{f}$ Converges to $P_{f}$.

The simulation results can be obtained from formula(15), it is clear that analog precision increases with the number of $N$.

Reliability analysis of discharge capacity of diversion structures, not only wants to get the single value of reliability, but also wants to know the effect of hydraulic factors on the reliability. In particular, it is necessary to analyse the various hydraulic factors mean-variance change impacting on reliability.

Its practical significance is self-evident, because of reducing hydraulic factors mean-variance deviation means reducing construction errors, which can be carried out to improve the construction quality of standard quantitative guidance, namely the decrease of mean-variance deviation causes reliability increase, among them once the most obvious hydraulic factors are found, thus the most critical factors of quality control in the construction are found.

Keeping the others constant, respectively let mean-variance deviation of the hydraulic radius $R$, tunnel slope rate $i$, tunnel length $L$, roughness $n$ occur some increase, the resulting change of discharge capacity reliability reflects the discharge capacity reliability sensitivity to changes with the influence of these factors.

\section{ENGINEERING EXAMPLES}

Being taken diversion tunnel of Lechang Gorge hydroelectric project for example, risk of its discharge capacity is analysed and calculated.

\section{A. Basic parameters of the diversion tunnel}

Elevation of upstream cofferdam crest is $120.8 \mathrm{~m}$, elevation of downstream cofferdam crest of $108.0 \mathrm{~m}$; elevation of imported floor of the diversion tunnel for $94.0 \mathrm{~m}$, elevation of export floor of the diversion tunnel for $92.0 \mathrm{~m}$, pressure segment length of tunnel for $572.0 \mathrm{~m}^{[11]}$.

Tunnel body section is shown in Fig. 1:

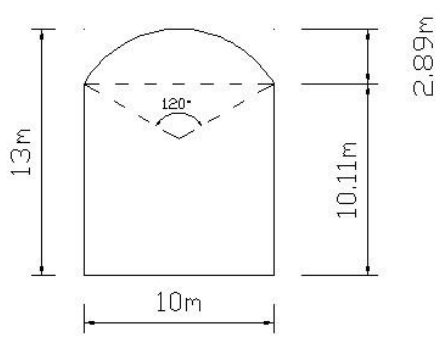

Figure 1. Diversion tunnel body section

Calculation parameters : $A=121.6 \mathrm{~m}^{2}, L=42.3 \mathrm{~m}$, $R=2.87 \mathrm{~m}, C=85.15$

Cofferdam retaining water standard using 10 year flood standard within 4months period (October-following march), the corresponding peak flow at the dam site is $1730.0 \mathrm{~m}^{3}$

\section{B. Selection of the original random variables}

According to the formula (4), the random variable is choosed:

Flow coefficient $\mu$ and elevation of upstream water level $H_{0}$ are random variables, approximately normally distributed; the mean of $\mu$ is 0.748 , mean-variance deviation of is $\mu 0.05$; the mean of $H_{0}$ is 119.0, mean-variance deviation of $H_{0}$ is $\mu 0.8$.

\section{Algorithms \& flow chart}

This example using $V B$ language program, flow chart as shown in Fig. 2, the basic steps are as follows:

1) $n$ random number of flow coefficient $\mu$ are generated, its mean is 0.748 , mean-variance deviation is 0.05 , and they are the normal distribution.

2) $n$ random number of elevation of upstream water level $H_{0}$ are generated, its mean is 119.0 , mean-variance deviation is 0.8 , and they are the normal distribution.

3) Above parameters are put into formula (4) to calculate(simulation), i.e. one time calculation is a test, if the calculated value is less than the amount of water $1730.0 \mathrm{~m}^{3}$, meaning failure, counter +1 ;

4) Repetition goes on until the end of trial; the ratio of the total number of failures and the total number of simulation is the failure probability.

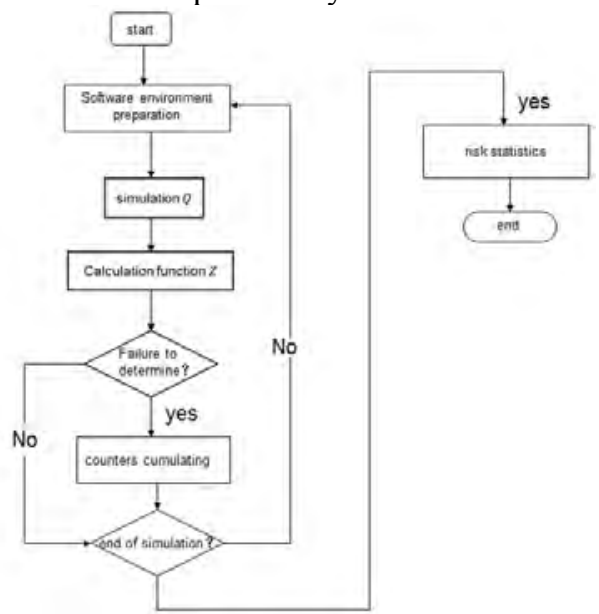

Figure 2. Simulation process of $M C$ method

\section{Calculated results}

when a simulating count 10,000 tests, failure probability $P_{f}=23.24 \%$; When the number increased to 20,000 times, $P_{f}=23.17 \%$; Again increased to 100,000 times, $P_{f}=23.18 \%$. It can be seen that with the increase of number of simulation, in particular more than 20,000 times, probability of failure $P_{f}$ tends to be stabilized.

The standard this diversion tunnel is 10 -years flood, using period is 3 years. The Yen B.C. classical probability formula ${ }^{[12]}$ is for reference, getting the risk $n=0.271$, compared with the calculated results of $M C$ method in this paper, the former is slightly larger

The Yen B.C. formula focuses on the influence of two factors on the risk: diversion standard (especially water frequency) as well as the service life of diversion structures, while this chapter focuses on the various factors of hydraulic uncertainty impacting on tunnel discharge 
capacity, the are two completely different angles. The results of different study point, can support each other, to some extent, the approach in this paper is reasonable.

Diversion tunnels were put into operation on August 2, 2009 , to November 2011, experienced a total of 6 times flows exceed peak test of $1000 \mathrm{~m}^{3}$, the diversion tunnel and import \& export structure remained stability. Practice shows that the reliability of diversion tunnel discharge capacity is in line with the actual requirements.

\section{CONCLUSION}

Research on discharge capacity reliability of construction diversion tunnel has practical significance, involving a new subject of study of diversion standards. Some objective and human factor would have an effect on reliability of tunnel discharge. With the proper analysis of random variable distribution types, proper statistical analysis, establishing a reasonable probability density functions and performance function, and rational choice of numerical simulation method, thus the research results tend to be more objective and actual status.

Study on discharge capacity reliability of diversion tunnel can provide a certain reference value for the future, especially with the diversion project of the same type and same river basin.

\section{REFERENCE}

[1] MI Ziming, ZHONG Denghua, and LIU Donghai, "'"Reliability analysis on discharge of diversion structure," Journal of Tianjin University,Vol.34,No.2, pp. 154-157, Mar.2001.

[2] $\mathrm{Hu}$ Zhigen, Liu Quan,Chen Zhiding, "Risk analysis of construction diversion”, Science Press, Beijing, 2010.

[3] Li Qijun, Chen Zhao, "Theory and application of dam overtopping risk", China water \& power press, 2008.

[4] Zhang Junzhi, "Existing engineering structure reliability theory and its application", China water \& power press, 2007.

[5] Zhao Guopan, "Engineering structure reliability theory and its application”, Dalian University of technology press,1996.

[6] Ma Rongyong, "Earth-rock dam risk analysis method and its application", Science Press, Beijing, 2004.

[7] Chu Xiangyuan, "Study on hydraulic model of uncertainty", Journal of Hydraulic Engineering, vol.5, pp. 33-38, 1992.

[8] Li Rong, "Discussion on the hydraulic factors influencing the Man-Ning roughness value $n$ ", Journal of Hydraulic Engineering, vol.12, pp. 62-68,1989.

[9] Wang Zhuofu, "Risk analysis of construction diversion", Journal of Hydraulic Engineering, vol.5, pp. 65-71, 1992.

[10] Xiong Guangleng, Xiao Tianyuan, Zhang Yanyun, "Continuous system simulation and discrete event system simulation", Tsinghua University Press, Beijing, 1991.

[11] Liu Lijie, Yao Limin, "Construction diversion design of Lechang Gorge hydroelectric project", Guangdong Water Resouces and Hydropower, vol.1, pp. 64-67, 2012.

[12] Yuan Guangyu, Hu Zhigen, "Hydraulic engineering construction", China water \& power press, pp.28, 2009 